\title{
Preliminary results of the Two Micron Galactic Survey
}

\author{
X. Calbet, T. Mahoney, P. Hammersley, F. Garzón and M. Selby \\ Instituto de Astrofísica de Canarias, E-38200 La Laguna, Tenerife (Spain)
}

\begin{abstract}
We present the first results of the Two-Micron Galactic Survey (TMGS). The sources are concentrated on the Galactic plane, the width of the distribution increasing between $0^{\circ}$ and $30^{\circ}$ longitude. Several obscuration lanes are seen, showing that $K$-band absorption can be high and patchy.
\end{abstract}

The aim of the TMGS is to map large areas of the Galactic plane and bulge (Mahoney et al. 1990). The TMGS, which was started in 1988, uses the $1.5 \mathrm{~m}$ Carlos Sánchez Telescope of the Observatorio del Teide (Tenerife). It uses the ICSTM Seven Channel Near-Infrared Camera, which has $7 \times 15 \operatorname{arcsec}^{2}$ detectors aligned in declination and a standard $K$ filter. The data are obtained by making drift scans, typically two hours long, across the Galactic plane. The limiting magnitude of $\approx$ +10.5 and is complete to $\approx+9.8$. The positional accuracy is currently 1 arcsec in RA and 12 arcsec in declination.

From the data reduced so far, which corresponds to the first four years of the project, we have detected 400000 sources and covered an area of $240 \mathrm{deg}^{2}$. The map shown in Figure 1 is of a region centred on $l=30^{\circ}, b=0^{\circ}$. A histogram of source counts and superimposed plot of $K-[60]$ against RA is given in Figure 2. Correlating this survey with IRAS data, it can be seen that objects at the centre of the plane or in one of these obscuration zones are more reddened.

\section{References}

Mahoney, T., Selby, M., Garzón, F., Hammersley, P. and Calbet, X 1990, in B. Jarvis and D. Terndrup (eds.) Bulges of Galaxies. ESO/CTIO Workshop held at La Serena, Chile 16-19 January 1990. 


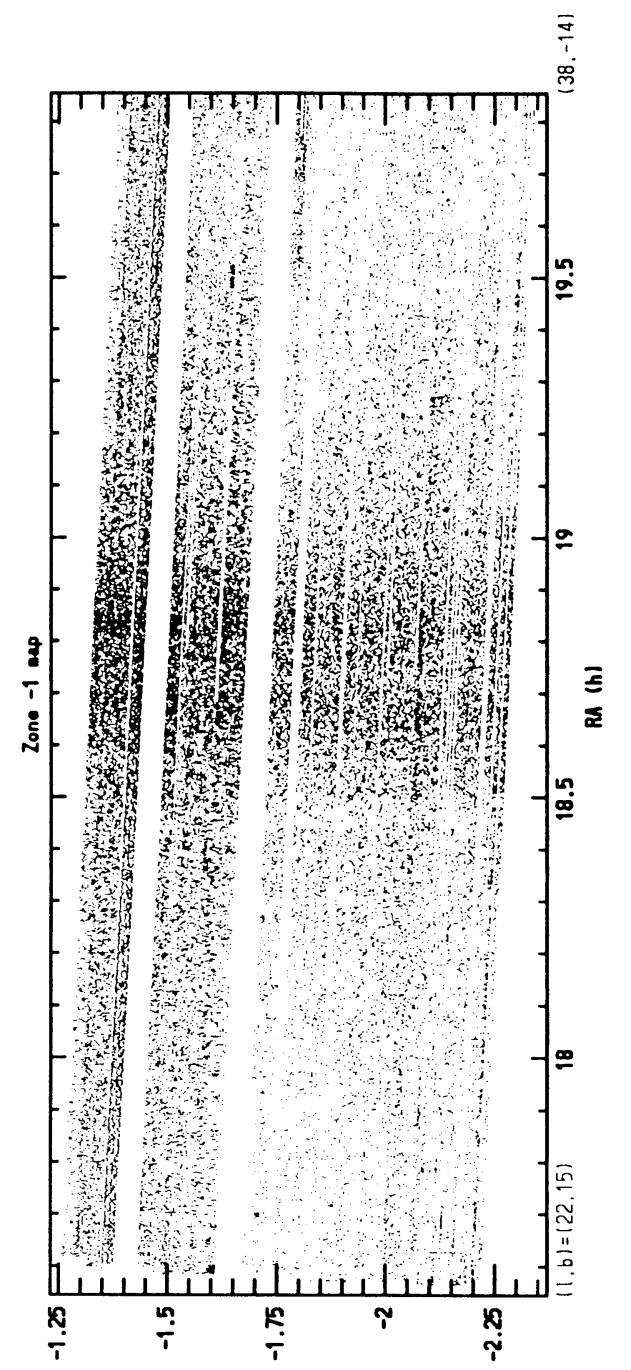

[ing J30

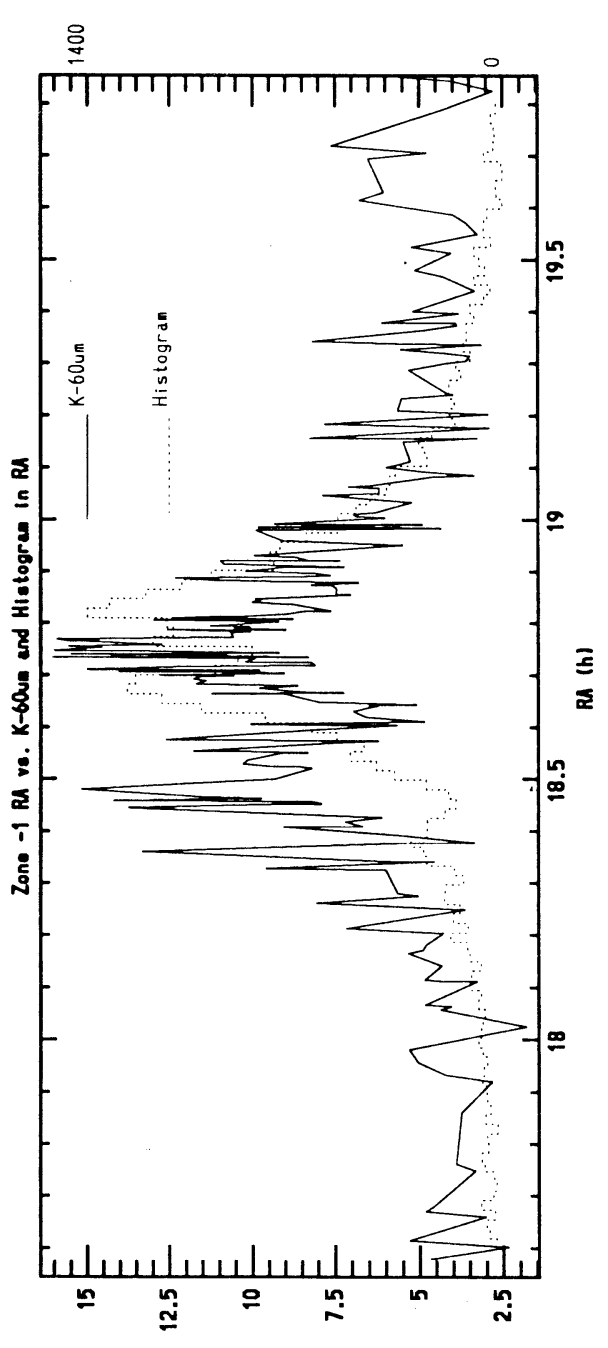

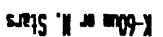

Figure 1 